\title{
NONCONTRACTIVE UNIFORMLY LIPSHITZIAN SEMIGROUPS IN HILBERT SPACE
}

\author{
DARYL TINGLEY ${ }^{1}$
}

\begin{abstract}
It is shown that any $k$-Lipshitzian, $k<\pi / 2$, noncontractive commutative semigroup acting on a closed bounded convex set in Hilbert space has a common fixed point.
\end{abstract}

1. Introduction. Let $\mathcal{F}=\left\{f_{\alpha} \mid \alpha \in A\right\}$ be a semigroup of mappings of a metric space $(M, d)$ into itself. The semigroup $\mathcal{F}$ is said to have a fixed point if there exists $x_{0} \in M$ with $f_{\alpha}\left(x_{0}\right)=x_{0}$ for all $\alpha \in A$, and $\mathcal{F}$ is said to be uniformly $k$-Lipshitzian if for each $x, y \in M$ and each $\alpha \in A$,

$$
d\left(f_{\alpha}(x), f_{\alpha}(y)\right) \leq k d(x, y) .
$$

$\mathcal{F}$ is said to be left reversible if every two right ideals of $\mathcal{F}$ have a nonempty intersection (i.e. for $f, g \in \mathcal{F}, f \mathcal{F} \cap g \mathcal{F} \neq \emptyset$ ). Commutative semigroups, and in particular $\left\{f^{n}: n=0,1, \ldots\right\}$ for some function $f$, are left reversible.

In [4] Goebel, Kirk, and Thele showed that if $X$ is a Banach space with $\delta(1)>0$ (where $\delta$ is the modulus of convexity function) then there is a constant $k_{0}^{\prime}>1$ such that any left reversible uniformly $k$-Lipshitzian semigroup $\mathcal{F}, k<k_{0}^{\prime}$, acting on a closed bounded convex set $K$ in $X$ has a fixed point. Clearly there is a maximum choice of $k_{0}^{\prime}$ which we call $k_{0}$. In [4] it was shown that for Hilbert space $(\mathcal{H}), \sqrt{5} / 2 \leq k_{0} \leq 2$. Downing and Ray [3] improved this estimate of $k_{0}$, for Hilbert space, by showing that $\sqrt{2} \leq k_{0}$ while in [1], Baillon has an example (presented here in $\S 2$ ) which shows that $k_{0} \leq \pi / 2$. (Lim [6 and 7] has improved the results of [4] for the $L^{p}$ spaces.)

In this note we show that under the additional assumption that $₹$ be a noncontractive (i.e. $\|x-y\| \leq\|f(x)-f(y)\|$ for each $f \in \mathcal{F}$ and all $x, y \in K$ ) uniformly $(\pi / 2-\delta)$-Lipshitzian $(\delta>0)$ commutative semigroup, then $\mathcal{F}$ has a fixed point. The example of Baillon, showing that $k_{0} \leq \pi / 2$, is noncontractive (this is proven in $\S 2$ of this paper), hence $\pi / 2$ is the exact value of $k_{0}$ in the case of noncontractive commutative semigroups. We note that the example in [4] showing that $k_{0} \leq 2$ is also noncontractive.

2. In this section we present Baillon's example $[\mathbf{1}]$ of a noncontractive uniformly $\pi / 2$-Lipshitzian semigroup of mappings of a closed bounded convex subset of $l^{2}$ which contains no fixed point.

Received by the editors June 1, 1983 and, in revised form, October 5, 1983.

1980 Mathematics Subject Classification. Primary 47H10.

Key words and phrases. Fixed point, uniformly $k$-Lipshitzian, noncontractive.

${ }^{1}$ The author would like to thank Professor M. Edelstein for his help and encouragement, and the referee for his careful reading of the manuscript, his proof that the example in $\S 2$ is noncontractive, and for pointing out that this example has previously been announced in [1].

(C) 1984 American Mathematical Society $0002-9939 / 84 \$ 1.00+\$ .25$ per page 
Let $S$ be the shift operator. That is, $S\left(\left(x_{1}, x_{2}, \ldots\right)\right)=\left(0, x_{1}, x_{2}, \ldots\right)$. Let $e_{1}=(1,0,0, \ldots)$ and $K=\left\{x \in l^{2}: x=\left(x_{1}, x_{2}, \ldots\right),\|x\| \leq 1, x_{i} \geq 0, i=1,2, \ldots\right\}$. Define $f$ by

$$
f(x)=\cos \left(\frac{\pi}{2}\|x\|\right) e_{1}+\sin \left(\frac{\pi}{2}\|x\|\right) \frac{S(x)}{\|x\|} .
$$

Several properties of $f$ are immediate,

$$
\begin{gathered}
\|f(x)\|=1 \quad \text { for all } x \in K . \\
\text { If }\|x\|=1 \quad \text { then } f(x)=S(x) . \\
f^{n}(x)=S^{n-1} f(x) \quad \text { (from 2.1 and 2.2). }
\end{gathered}
$$

If $f(x)=x(x \in K)$ then $x=f^{2}(x)=S f(x)=S(x)$. Since $S(x)=x$ implies that $x=0$, and $f(0)=(1,0, \ldots), f$ has no fixed points. Once it is estabished that

$$
\|x-y\| \leq\|f(x)=f(y)\|<\pi / 2\|x-y\|, \quad x, y \in K,
$$

the semigroup $\mathcal{F}=\left\{f^{n} \mid n=1,2, \ldots\right\}$ is readily seen to be noncontractive uniformly $\pi / 2$-Lipshitzian with no fixed points. Note that the second inequality of 2.4 is strict, so $\pi / 2$ is a strict Lipshitz constant for $\mathcal{F}$.

The fact that $\mathcal{F}$ is noncontractive has not been mentioned in the literature, we believe, and as it shows that the constant $\pi / 2$ in our main theorem is the "best possible", we present a brief proof (due to the referee).

PROOF THAT $\mathcal{f}$ IS NONCONTRACTIVE. We readily compute that

$$
\begin{aligned}
\|f(x)-f(y)\|^{2}-\| & -y \|^{2}=\left(\cos \left(\frac{\pi}{2}\|x\|\right)-\cos \left(\frac{\pi}{2}\|y\|\right)\right)^{2} \\
& +\left\|\frac{x}{\|x\|} \sin \left(\frac{\pi}{2}\|x\|\right)-\frac{y}{\|y\|} \sin \left(\frac{\pi}{2}\|y\|\right)\right\|^{2}-\|x-y\|^{2} \\
= & 2-2 \cos \left(\frac{\pi}{2}\|x\|\right) \cos \left(\frac{\pi}{2}\|y\|\right)-\left(\|x\|^{2}+\|y\|^{2}\right) \\
& +2\left\langle\frac{x}{\|x\|}, \frac{y}{\|y\|}\right\rangle\left\{\|x\|\|y\|-\sin \left(\frac{\pi}{2}\|x\|\right) \sin \left(\frac{\pi}{2}\|y\|\right)\right\} .
\end{aligned}
$$

The well-known inequality $\sin t \geq 2 t / \pi$ (for $0 \leq t \leq \pi / 2$ ) shows that the expression in curly brackets is less than or equal to 0 , thus replacing $\langle x /\|x\|, y /\|y\|\rangle$ by one, we get

$$
\begin{aligned}
\|f(x)-f(y)\|^{2}-\|x-y\|^{2} & \geq 2-2 \cos \frac{\pi}{2}(\|x\|-\|y\|)-(\|x\|-\|y\|)^{2} \\
& =4 \sin ^{2} \frac{\pi}{4}(\|x\|-\|y\|)-(\|x\|-\|y\|)^{2} .
\end{aligned}
$$

A final appeal to $\sin t \geq 2 t / \pi$ shows that this is nonnegative.

3. In this section $H$ shall denote a Hilbert space, $K$ a closed bounded convex set of $\mathcal{H}, S$ a subset of $K, f: K \rightarrow K$ a noncontractive function, and $\mathcal{F}: K \rightarrow K$ a noncontractive commutative semigroup. We show that if $\mathcal{F}$ is uniformly $(\pi / 2-\delta)$ Lipshitzian, $\delta>0$, then $\mathcal{F}$ has a fixed point.

Let $S \subseteq K$. Then $S$ is bounded, as $K$ is. For $x \in \mathcal{H}$ define

$$
r(S, x)=\sup \{\|x-s\|: s \in S\},
$$




$$
r(S)=\inf \{r(S, x): x \in \mathcal{H}\},
$$

and let $c(S)$ be the unique point of $\mathcal{H}$ such that $r(S, c(S))=r(S)$. Equivalently, $c(S)$ is the unique point of $\mathcal{H}$ such that $\bar{B}(c(S), r(S)) \supseteq S$ (where $\bar{B}(x, r)$ denotes the closed ball about $x$ of radius $r$ ). The point $c(S)$ is called the Chebyshev center of $S$. When no confusion arises, let $r(x)=r(S, x), r=r(S)$ and $c=c(S)$. It is well known and easily shown (cf. [5]) that $c(S)$ lies in the closed convex hull of $S$ (denoted $\overline{c o}(S)$ ) and hence is in $K$.

LEMma 3.1. For $x \in \mathcal{H}, r^{2}+\|c-x\|^{2} \leq r^{2}(x)$.

Proof. To simplify notation, assume that $c=0$. Then $\|y\| \leq r$ for all $y \in S$. For a contadiction, suppose that

$$
\varepsilon=\frac{r^{2}+\|x\|^{2}-r^{2}(x)}{2\|x\|}>0
$$

and let $z=\varepsilon x /\|x\|$. We claim, then, that $r^{2}(z) \leq r^{2}-\varepsilon^{2}$. To see this let $y \in \mathcal{S}$, and consider the two cases $\langle y, x /\|x\|\rangle>\varepsilon$ and $\langle y, x /\|x\|\rangle \leq \varepsilon$. In the first case a straightforward calculation shows that $\|y-z\|^{2} \leq r^{2}-\varepsilon^{2}$. For the second case the cosine law shows that

$$
\|y-z\|^{2}=\|y-x\|^{2}-\|x-z\|^{2}+2\langle y-z, x-z\rangle .
$$

It can now be shown that $\langle y-z, x-z\rangle \leq 0$, hence

$$
\|y-z\|^{2} \leq r^{2}(x)-\|x-z\|^{2} .
$$

Using the definition of $\varepsilon$ this then yields

$$
\|y-z\|^{2} \leq r^{2}-\varepsilon^{2} .
$$

Thus $r^{2}(z) \leq r^{2}-\varepsilon^{2}$. However this contradicts the definition of $c$, and hence the lemma has been established.

Lemma 3.2. For every $S \subseteq K, \sup \{r(T): T \subset S$ and $T$ is finite $\}=r$.

Proof. Assume there is an $r^{\prime}<r$ such that $r(T)<r^{\prime}$ for every finite set $T \subset S$. The finite intersection property then shows that $\bigcap_{x \in S} \bar{B}\left(x, r^{\prime}\right) \neq \emptyset$, implying that $r=r(s) \leq r^{\prime}$ a contradiction.

THEOREM 3.3 (KIRSZBRAUN). Let $\left\{x_{i}: i \in I\right\}$ and $\left\{y_{i}: i \in I\right\}$ be sets in $\mathcal{H}$ and $\left\{r_{i}: i \in I, r_{i}>0\right\}$ be a set of real numbers such that

$$
\left\|x_{i}-x_{j}\right\| \leq\left\|y_{i}-y_{j}\right\| \quad(i, j \in I) .
$$

Then

$$
\bigcap_{i \in I} \bar{B}\left(x_{i}, r_{i}\right)=\emptyset \quad \text { implies } \bigcap_{i \in I} \bar{B}\left(y_{i}, r_{i}\right)=\emptyset .
$$

Proof. [8, p. 47].

Lemma 3.4. For each $S \subseteq K, r(S) \leq r(f(S))$.

Proof. For a contradiction, assume that $\varepsilon=r(S)-r(f(S))>0$. By the definition of $r(S)$,

$$
\bigcap_{s \in S} \bar{B}(s, r(S)-\varepsilon)=\emptyset .
$$


As $f$ is noncontractive,

$$
\left\|f\left(s_{1}\right)-f\left(s_{2}\right)\right\| \geq\left\|s_{1}-s_{2}\right\| \text { for all } s_{1}, s_{2} \in S .
$$

Kirszbraun's theorem implies that

$$
\emptyset=\bigcap_{s \in S} \bar{B}(f(s), r(S)-\varepsilon)=\bigcap_{s \in S} \bar{B}(f(S), r(f(S)))=\{c(f(S))\}
$$

leading to a contradiction.

LEMMA 3.5. Assume that $S \subseteq K$ satisfies $f(S) \subseteq S$. Then $r(S)=r(f(S))$ and $c(S)=c(f(S))$.

Proof. Because $f(S) \subseteq S$ we have $r(f(S)) \leq r(S)$. On the other hand Lemma 3.4 shows that $r(S) \leq r(f(S))$. Hence $r(S)=r(f(S))$. Now

$$
f(S) \subseteq S \subseteq \bar{B}(c(S), r(S))=\bar{B}(c(S), r(f(S)))
$$

However $c(f(S))$ is the unique point in $\nLeftarrow$ such that $\bar{B}(c(f(S)), r(f(S))) \subseteq f(S)$. Thus $c(S)=c(f(S))$.

LEMmA 3.6. Let $S \subseteq K$ satisfy $f(S) \subseteq S$. Then for each $x \in K, \| f(x)-$ $c(S)\|\geq\| x-c(S) \|$.

Proof. From Lemma 3.5, $c(S)=c(f(S))$ and $r(S)=r(f(S))$. Letting $c=$ $c(S)$ and $r=r(S)$, assume for some $x \in K$ that $\|x-c\|>\|f(x)-c\|$. Let $\varepsilon=\|x-c\|-\|f(x)-c\|$. Now $\bigcap_{s \in S} \bar{B}(s, r)=\{c\}$, so

$$
\phi=\bigcap_{s \in S} \bar{B}(s, r) \cap \bar{B}(x,\|x-c\|-\varepsilon) .
$$

By Kirszbraun's Theorem (Theorem 3.3)

$$
\phi=\bigcap_{s \in S} \bar{B}(f(s), r) \cap \bar{B}(f(x),\|f(x)-c\|)=\{c\} .
$$

Thus we have a contradiction, hence

$$
\|x-c\| \leq\|f(x)-c\|
$$

If $\mathcal{F}=\left\{f_{\alpha}: \alpha \in A\right\}$ is a commutative (or left reversible) semigroup then the set $A$ can be directed as follows. For $\alpha, \beta \in A$ define

$$
\alpha \leq \beta \text { if and only if } f_{\alpha} \varsubsetneqq \supseteq f_{\beta} \varsubsetneqq .
$$

Thus for each $x, \mathcal{F}(x)=\left\{f_{\alpha}(x): \alpha \in A\right\}$ is a net. Also, without loss of generality when finding fixed points, it may be assumed the identity is in $\mathcal{F}$.

COROLLARY 3.7. If $₹: K \rightarrow K$ is a commutative semigroup of noncontractive mappings, $K$ a closed bounded convex set in $\mathcal{H}$, and $\mathcal{F}: S \subseteq K \rightarrow S$ then for $\alpha \geq \beta$,

$$
\left\|f_{\alpha}(x)-c(S)\right\| \geq\left\|f_{\beta}(x)-c(S)\right\|
$$

for each $x \in K$.

Proof. Because $f_{\alpha} ₹ \supseteq f_{\beta} \mathcal{F}$, and the identity is in $\mathcal{F}, f_{\beta}=f_{\alpha} f_{\eta}, \eta \in A$, and as $\mathcal{F}$ is commutative, $f_{\beta}(x)=f_{\eta}\left(f_{\alpha}(x)\right)$. The corollary follows from Lemma 3.6.

REMARK. This corollary is the only point in our argument where the commutativity of $\mathcal{F}$ is used. If $\mathcal{F}$ were right-reversible, rather than commutative, the corollary would be valid. 
LEMMA 3.8. For every $\varepsilon>0$ there is a finite subset $T \subseteq S$ such that $\| c(S)-$ $c(f(T)) \|<\varepsilon$ for every noncontractive function $f$ with $f(S) \subseteq S$.

Proof. Let $\varepsilon>0$ be given. Lemma 3.2 shows that there is a finite subset $T \subseteq S$ with $r^{2}(S)-r^{2}(T)<\varepsilon^{2}$. Let $f$ be an arbitrary noncontractive function with $f(S) \subseteq S$. From Lemma 3.1, we have

$$
r^{2}(f(T))+\|c(f(T))-c(S)\|^{2} \leq r^{2}(f(T), c(S)) \leq r^{2}(S) .
$$

Lemma 3.4 shows that $r(T) \leq r(f(T))$, so

$$
r^{2}(T)+\|c(S)-c(f(T))\|^{2} \leq r^{2}(S) .
$$

Hence $\|c(S)-c(f(T))\|<\varepsilon$, establishing the lemma.

LEMMA 3.9. Let $\varepsilon>0$ be given. Then there is a finite subset $T \subseteq S$ such that for every linear functional $h,\|h\|=1$, and each noncontractive function $f$ satisfying $f(S) \subseteq S$ it is true that for some $x \in T$,

$$
h(f(x))-h(c(S))<\varepsilon .
$$

ProOF. By Lemma 3.8 there is a finite subset $T \subseteq S$ such that for every noncontractive function $f,\|c(S)-c(f(T))\|<\varepsilon$. As has been mentioned, $c(f(T)) \in$ $\overline{\mathrm{co}}(f(T))$, hence for some $x \in T, h(x)-h(c(f(T))) \leq 0$. Thus

$$
h(x)-h(c(S)) \leq h(c(f(T)))-h(c(S))<\varepsilon .
$$

By an arc $\gamma$ in $\mathcal{H}$ (or any metric space) we mean the image of a function $\gamma:[a, b] \rightarrow$ $\mathcal{H}$ for some interval $[a, b]$ of $\mathbf{R}$. The length of an arc may be defined by purely metric means, without any differentiability assumption. We refer the reader to a book on metric geometry such as [2] for a discussion of arcs and their lengths. Let $l(\gamma)$ denote the arclength of (the image of) $\gamma$. If $f$ satisfies

$$
k_{1}\|x-y\| \leq\|f(x)-f(y)\| \leq k_{2}\|x-y\|
$$

and $\gamma$ lies in the domain of $f$, then $f \circ \gamma$ is an arc and

$$
k_{1} l(\gamma) \leq l(f(\gamma)) \leq k_{2} l(\gamma)
$$

LEMMA 3.10. Let $\gamma(t), a \leq t \leq b$ be an arc satisfying

$$
\frac{\langle\gamma(a), \gamma(b)\rangle}{\|\gamma(a)\|\|\gamma(b)\|} \leq \cos \theta, \quad 0 \leq \theta \leq \pi,
$$

and $\|\gamma(t)\| \geq d, t \in[a, b]$. Then the length of $\gamma$ is at least $d \cdot \theta$.

ProOF. Let $P$ be the projection of $\mathcal{H} \backslash\{0\}$ onto the sphere of radius $d$, defined by $P(x)=d \cdot x /\|x\|$. Certainly $P$ is nonexpansive on $\{x \in \mathcal{H}:\|x\| \geq d\}$ so $l(P(\gamma)) \leq l(\gamma)$. However $P(\gamma)$ is no shorter than the geodesic on $S$ joining $P(\gamma(a))$ to $P(\gamma(b))$, whose length is at least $d \cdot \theta$.

THEOREM. Let $\mathcal{F}=\left\{f_{\alpha} \mid \alpha \in A\right\}$ be a commutative semigroup (with identity) of self-mappings of a closed bounded convex set $K$ in a Hilbert space $\nVdash$. Assume that for some $\delta>0$,

$$
\|x-y\| \leq\left\|f_{\alpha}(x)-f_{\alpha}(y)\right\| \leq(\pi / 2-\delta)\|x-y\| .
$$

Then $\mathcal{F}$ has a fixed point. 
ProOF. Let $x_{0} \in K$ be arbitrary, $x_{\alpha}=f_{\alpha}\left(x_{0}\right)$ and $S=\left\{x_{\alpha} \mid \alpha \in A\right\}$. Note that $f_{\alpha}(S) \subseteq S$ for all $\alpha \in A$. Let $c=c(S), r=r(S)$. As mentioned earlier, $c \in$ $\overline{\operatorname{co}}\left\{x_{\alpha}: \alpha \in A\right\} \subseteq K$ (see [5]). Let $\left[c, x_{\alpha}\right]$ denote the arc $\left\{y: y=\lambda(c)+(1-\lambda) x_{\alpha}, 0 \leq\right.$ $\lambda \leq 1\}$. We now show that for some $x \in \bigcup_{\alpha \in A}\left[c, x_{\alpha}\right],\left\|c-f_{\alpha}(x)\right\| \leq(1-\delta / \pi) r$, for all $\alpha \in A$.

Clearly if $r=0$, then $x$ may be taken to be $c$ (which is a fixed point of $\mathcal{F}$ ). Thus, for a contradiction, assume that $r>0$ and that $\sup \left\{\left\|c-f_{\alpha}(x)\right\|: \alpha \in A\right\}>$ $(1-\delta / \pi) r$ for all $x \in \bigcup_{\alpha \in A}\left[c, x_{\alpha}\right]$.

Let $\varepsilon>0$ be given. By Lemma 3.9 choose a finite set $T \subseteq S$ such that for any $\alpha \in A$, and any linear functional $h,\|h\|=1$, there is a $z \in T$ with $h\left(f_{\alpha}(z)\right)-h(c)<$ $\varepsilon$. Since $\bigcup_{y \in T}[c, y]$ is compact, Corollary 3.7 implies that for some $\eta \in A$ and for every $x \in \bigcup_{y \in T}[c, y]$,

Define

$$
\left\|c-f_{\eta}(x)\right\|>\left(1-\frac{\delta}{\pi}\right) r .
$$

$$
h(x)=\left\langle\frac{f_{\eta}(c)-c}{\left\|f_{\eta}(c)-c\right\|}, x\right\rangle
$$

and let $z \in T$ satisfy $h\left(f_{\eta}(z)\right)-h(c)<\varepsilon$. Thus

$$
\left\langle\frac{f_{\eta}(c)-c}{\left\|f_{\eta}(c)-c\right\|}, \frac{f_{\eta}(z)-c}{\left\|f_{\eta}(z)-c\right\|}\right\rangle<\frac{\varepsilon}{(1-\delta / \pi) r} .
$$

Hence angle $f_{\eta}(c) c f_{\eta}(z)$ is greater than $\cos ^{-1}(\varepsilon /(1-\delta / \pi) r)$ and by Lemma 3.10

$$
l\left(f_{\eta}[c, z]\right)>\left(1-\frac{\delta}{\pi}\right) r \cos ^{-1}\left(\frac{\varepsilon}{(1-\delta / \pi) r}\right) .
$$

As $\varepsilon>0$ was arbitrary, it must be that

$$
\sup \left\{l\left(f_{\eta}[c, z]\right): \eta \in A, z \in S\right\} \geq\left(\frac{\pi}{2}-\frac{\delta}{2}\right) r .
$$

However, as $\left\|f_{\eta}(x)-f_{\eta}(y)\right\| \leq\left(\frac{\pi}{2}-\delta\right)\|x-y\|$ for all $x, y \in K$ and $\eta \in A$, then for each $\eta \in A$ and $z \in S$,

$$
l\left(f_{1}[c, z]\right) \leq\left(\frac{\pi}{2}-\delta\right)\|c-z\| \leq\left(\frac{\pi}{2}-\delta\right) r .
$$

Combining 3.3 and 3.4, we reach the desired contradiction, and conclude that for some $x \in \bigcup_{\alpha \in A}\left[c, x_{\alpha}\right]$,

$$
\left\|c-f_{\alpha}(x)\right\| \leq\left(1-\frac{\delta}{\pi}\right) r \quad \text { for all } \alpha \in A .
$$

Let $y_{0} \in K$ be arbitrary and let $r=r\left(\left\{f_{\alpha}\left(y_{0}\right): \alpha \in A\right\}\right)$. Assume that for $n<k, y_{n}$ has been defined so that

$$
\left\|y_{n}-y_{n-1}\right\| \leq 2\left(1-\frac{\delta}{\pi}\right)^{n-1} r
$$

and

$$
r\left\{f_{\alpha}\left(y_{n}\right): \alpha \in A\right\} \leq\left(1-\frac{\delta}{\pi}\right)^{n} r .
$$


Using the above argument with $x_{0}=y_{k-1}$ we find a point $y_{k}=x$ such that

$$
\left\{f_{\alpha}\left(y_{k}\right): \alpha \in A\right\} \subseteq \bar{B}\left(c\left(\left\{f_{\alpha}\left(y_{k-1}\right): \alpha \in A\right\}\right),\left(1-\frac{\delta}{\pi}\right)^{k} r\right)
$$

and hence (3.6) is satisfied with $n=k$, and

$$
\left\|y_{k}-c\left\{f_{\alpha}\left(y_{k-1}\right): \alpha \in A\right\}\right\| \leq\left(1-\frac{\delta}{\pi}\right)^{k} r \leq\left(1-\frac{\delta}{\pi}\right)^{k-1} r
$$

(recall we assume that $\mathcal{F}$ contains the identity). Also,

$$
\left\|y_{k-1}-c\left\{f_{\alpha}\left(y_{k-1}\right): \alpha \in A\right\}\right\| \leq r\left(\left\{f_{\alpha}\left(y_{k-1}\right): \alpha \in A\right\}\right) \leq\left(1-\frac{\delta}{\pi}\right)^{n-1} r .
$$

Hence 3.8 and 3.9 together show 3.5 is satisfied with $n=k$.

We conclude, using the continuity of the $f_{\alpha}$, that $\left\{y_{n}\right\}$ is a Cauchy sequence, and its limit is a fixed point of $\mathcal{F}$.

REMARK. The above theorem holds for a right reversible, rather than commutative, semigroup. See the remark following Corollary 3.6.

\section{REFERENCES}

1. J. B. Baillon, Quelques aspects de la théorie des points fixes dans les espaces de Banach. I, Séminaire d'Analyse Fonctionnelle de l'Ecole Polytechnique, no. VII, 1978-1979.

2. L. M. Blumenthal, Theory and applications of distance geometry, Oxford Univ. Press, London, 1953.

3. D. J. Downing and W. O. Ray, Uniformly Lipshitzian semigroups in Hilbert space, Canad. Math. Bull. 25 (1982), 210-213.

4. K. Goebel, W. A. Kirk and R. L. Thele, Uniformly Lipshitzian families of transformations in Banach spaces, Canad. J. Math. 26 (1974), 1245-1256.

5. V. Klee, Circumspheres and inner products, Math. Scand. 8 (1980), 363-370.

6. T. C. Lim, Fixed point theorems for uniformly Lipshitzian mappings in $L^{p}$ spaces, J. Nonlinear Analysis (to appear).

7. Some inequalities and their applications to fuxed point theorems of uniformly Lipshitzian mappings in $L^{p}$ spaces, preprint.

8. J. H. Wells and L. R. Williams, Embeddings and extensions in analysis, Springer-Verlag, Berlin, 1975.

\section{Department of Mathematics, Dalhousie University, Halifax, Nova Scotia, CANADA}

Current address: Department of Mathematics, Mount Allison University, Sackville, New Brunswick, Canada 